\title{
An Experimental Study on Heat Transfer Enhancement of Flat Plates Using Dimples
}

\author{
Amjad Khan ${ }^{1}$, Mohammed Zakir Bellary ${ }^{1}$, Mohammad Ziaullah ${ }^{1}$, Abdul Razak Kaladgi ${ }^{2}$, \\ ${ }^{1}$ Department of Electronics and communication Engineering, P.A College of Engineering, Mangalore, Karnataka, India \\ ${ }^{2}$ Department of Mechanical Engineering, P.A College of Engineering, Mangalore, Karnataka, India
}

Email address:

amjadece@pace.edu.in (A. Khan), mdzakir87@gmail.com (M. Z. Bellary), mdziya1990@gmail.com (M. Ziaullah), abdulkaladgi@gmail.com (A. R. Kaladgi)

\section{To cite this article:}

Amjad Khan, Mohammed Zakir Bellary, Mohammad Ziaullah, Abdul Razak Kaladgi. An Experimental Study on Heat Transfer Enhancement of Flat Plates Using Dimples. American Journal of Electrical Power and Energy Systems. Vol. 4, No. 4, 2015, pp. 34-38.

doi: $10.11648 /$ j.epes.20150404.11

\begin{abstract}
Dimples play a very important role in heat transfer enhancement of electronic cooling systems. In the current paper, the fluid flow and heat transfer characteristics of spherical dimples at different angle of orientation from the centre with apex facing the inlet were investigated. The experiment was carried out for laminar Natural convection conditions with air as a working fluid. The overall Nusselt numbers and heat transfer coefficient at different orientation angle of dimples were obtained. From the obtained results, it was observed that the Nusselt numbers and heat transfer coefficient increases with decrease in the orientation angle of dimples.
\end{abstract}

Keywords: Electronic Cooling, Natural Convection, Dimples, Passive Techniques

\section{Introduction}

The development of integrated electronic devices with increase level of miniaturization, higher performance and output has increased the cooling requirement of chips considerably. Also, as the chip temperature increases, the stability and efficiency issues increases and the problem of heat dissipation becomes a bottleneck for the development of chips in the electronic industry [1]. Passive heat transfer enhancement techniques are recently used in electronic industries to overcome these issues. In these techniques concavities (dimples), extended surfaces or fins, and protrusions are made on the flat plates to increase the heat transfer rate. Among these, the dimples i.e. concavities are considered important because they not only increase the heat transfer rate but also provide minimum pressure losses (important for pumping power requirements [1]). Dimples increases the heat transfer rate because they produce vortex pairs, increases turbulent mixing, delays growth of the boundary layer, creates reattachment zones necessary for the heat transfer enhancement. And as they do not project into the flow so they contribute less to the foam drag, to produce minimum pressure drop penalties [2]. Another advantage is that in dimple manufacture the removal of material takes place which reduces the cost and weight of the equipments.

Kuethe [3] can be considered as the first person to use dimples on flat surfaces. He observed that the dimples acts as vortex generator, produces turbulent mixing in the flow \& increases the heat transfer rate. Afanasyev et al [4] conducted an experiment on friction and heat transfer analysis on flat plates using spherical dimples. And observed an increment of $30-40 \%$ in the heat transfer rate. No significant effect on the hydrodynamics of flow was observed in their study. Chyu et al [5] used tear drop type dimples along with hemispherical dimples to study the heat transfer characteristics of air flow in the channel. They observed a considerable increment in the heat transfer rate for the surfaces having dimples (About 2.5 times then their smooth surfaces). Mahmood et al [6] conducted an experiment to investigate the effect of dimples on heat transfer using the flow visualization techniques and concluded that the periodic nature of shedding off of vortices is the main cause of enhancement of heat transfer (much more pronounced at the downstream rims of the dimples). Mahmood et al [7] conducted an experiment to study the effect of Reynolds number, aspect ratio, and temperature ratio in a channel with dimples. They observed through the 
flow visualization techniques that the secondary vortices that are shed off from the dimples become stronger as the nondimensional channel height to dimple diameter (H/D) ratio decreases and increases the local Nusselt number in these regions. Rao et al [8] conducted an experiment to investigate the effect of dimple depth on flow and heat transfer characteristics in a pin fin-dimple channel and observed that compared to the pin fin channel, the pin fin dimple channels have improved convective heat transfer performance and especially the pin fin-dimple channel with dimples of higher depth have higher values of Nusselt number.

From the literature review, it is very much clear that dimples have high potential to enhance the heat transfer rate, together with producing minimum pressure drop penalties. The other advantages include low weight, low cost and low fouling rates [9]; however, much of the research either numerical or experimental is on spherical dimples of uniform diameter $[4,10]$. And most of the research is confined to flow in the channel or Internal flow (using forced convection), with a very few studies on external flow [10].So the main focus of this study is to investigate the effect of spherical dimples on aluminum/copper test plates under laminar natural flow conditions.

\section{Experimental Setup}

The fabricated experimental setup used in this study is as shown in the figure below

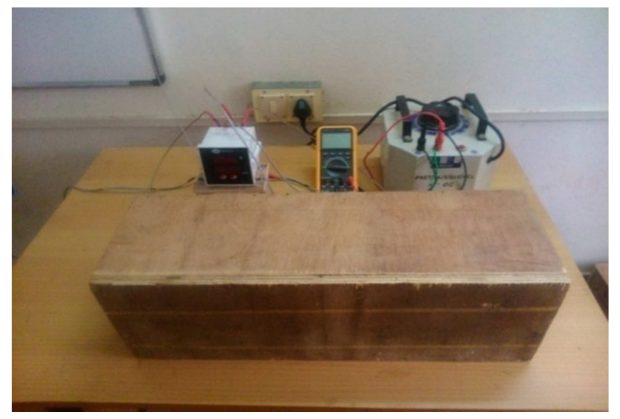

Figure 1. Experimental setup.

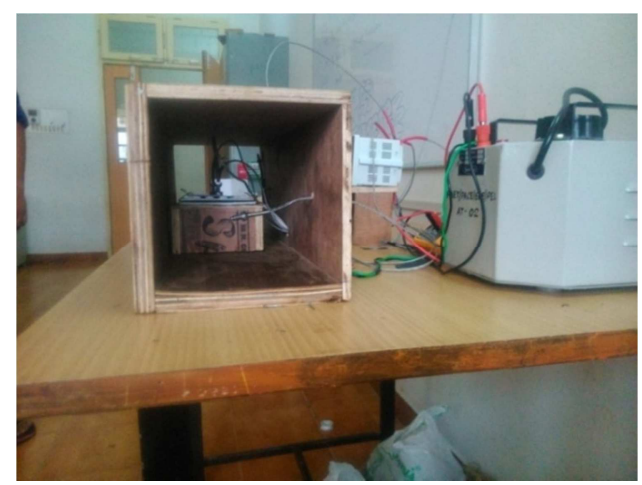

Figure 2. Experimental setup (side view).

It consist of a heater with a capacity of 200 watts, Dimmer stat, Digital temperature, voltmeter and ammeter with $\mathrm{J}$ type thermo couple. The test plates were placed on heater and a constant heat flux is supplied through dimmer stat to heater. Air flows parallel to the dimpled test surface. The plate heater is fixed at the bottom of the test plate and was connected to power socket through dimmer stat. Dimmer stat readings were varied to give the required heat input to the test plate. Only top dimpled surface of the test plate was exposed to the air stream from which the convective heat transfer to the air stream would takes place. After reaching a steady state, surface temperature of plate $\&$ air temperature were measured with the help of thermocouple.

Table 1. Components and Specifications.

\begin{tabular}{ll}
\hline Components & Specification \\
\hline Heater & $100 \mathrm{~W}, 4$ "x4" \\
Dimmer stat & $6 \mathrm{~A}, 230 \mathrm{~V}$ \\
Digital Temperature Indicator & 6 channel,12000C, 230V \\
Casing & A wooden casing of size of 8"x8" and \\
& 2feet long. \\
Thermocouple & K-Type, 3000C, 1m long. \\
Digital Multi-Meter & Voltmeter, Ammeter \\
Test plate & $10 \times 10 \times 2 \mathrm{~cm}$ aluminium/copper plates \\
\hline
\end{tabular}

\section{Results and Discussion}

Experiments were conducted on aluminum/copper test plates with spherical dimples of non uniform diameters made on flat plates. The different arrangements of dimples used are

Case a. Aluminum plate Dimples with $30^{\circ}$ orientations with centerline \& apex facing the inlet.

\section{ALUMINUM PLATE (AT 30)}

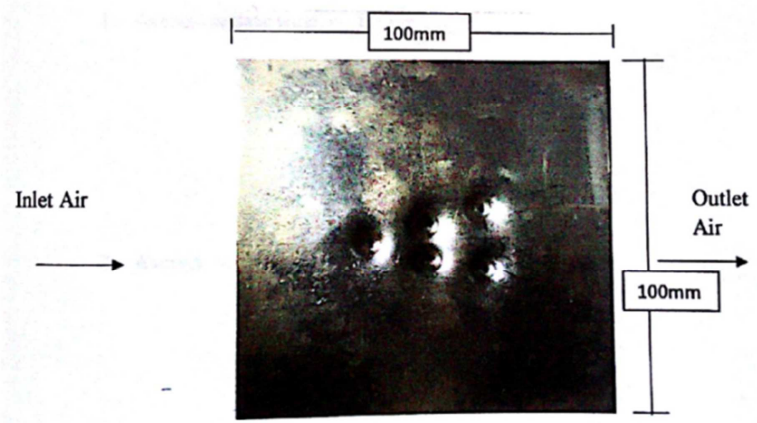

Case b. Dimples with $60^{\circ}$ orientations with centerline \& apex facing the inlet.

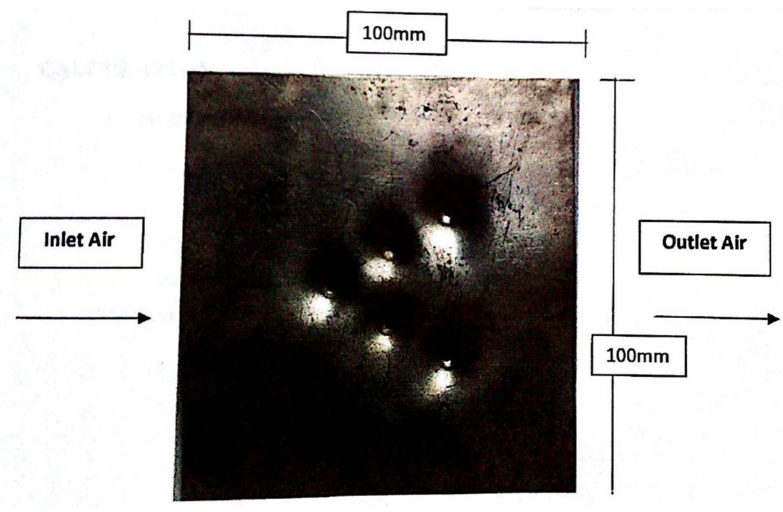


Case c. Dimples with $90^{\circ}$ orientations with centerline \& apex facing the inlet.

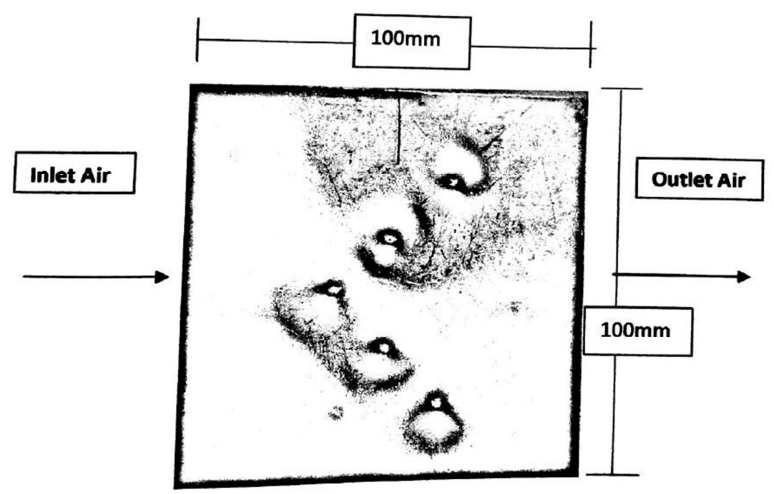

The data obtained were used to find important heat transfer parameters like Nusselt number, heat transfer coefficient, and heat transfer rate. And the experimental findings have been plotted in the form of graphs, mainly

- Nusselt number(Nu) vs. Reynolds number(Re)

- Heat transfer coefficient (h) vs. Reynolds number(Re)

- Heat transfer rate Q vs. Reynolds number(Re)

Figure 3, 4, 5 shows variation of Nusselt number ' $\mathrm{Nu}$ ', heat transfer coefficient ' $h$ ' and heat transfer rate ' $Q$ ' with different orientation of dimples. It can be seen that as expected the Nusselt number, heat transfer coefficient ' $h$ ' and heat transfer rate ' $Q$ ' increases with decrease in orientation angle of dimples. The increase in the Nusselt number as compared to flat plate may due to direct flow impingement on the downstream boundary of the plate and strengthened flow mixing of vortices at the downstream of the plate $[1,11]$ The formation of vortex pairs, periodically shedding off from the dimples at low angles, a large up wash regions with some fluids coming out from the central regions of the dimples, are the main causes of enhancement of Nusselt number [6].This happens mostly at low angle orientation of dimples $\left(30^{\circ}\right)$, as at low angles the dimples are very close to each other so a pronounce effect of dimples is felt. It can also be seen that the variation in the Nusselt number is almost gradual with orientation as expected $[12,13]$.

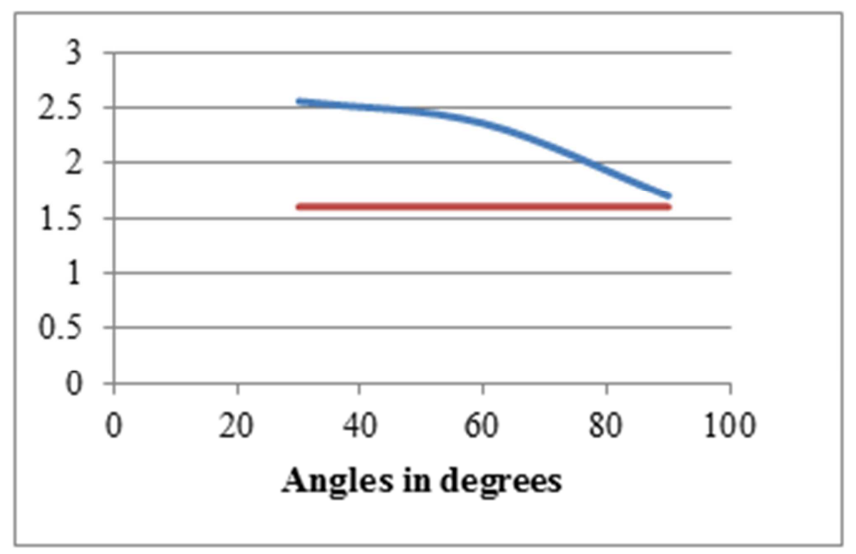

Figure 3. Variation of Nusselt number with orientation of dimples.

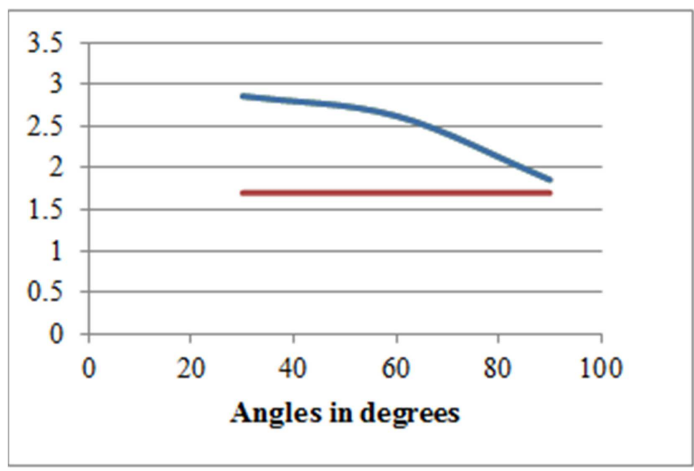

Figure 4. Variation of heat transfer coefficient with orientation of dimples.

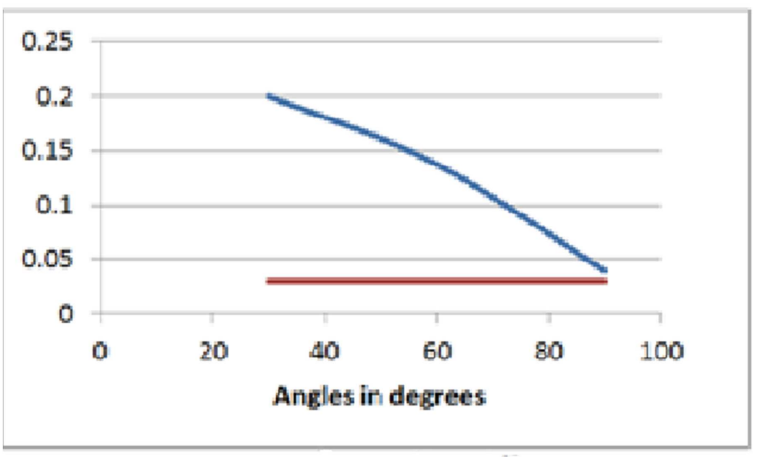

Figure 5. Variation of heat transfer rate with orientation of dimples.

Figure $6,7,8$ shows variation of Nusselt number ' $\mathrm{Nu}$ ', heat transfer coefficient ' $h$ ' and heat transfer rate ' $Q$ ' with different orientation of dimples. It can be seen that as expected the Nusselt number, heat transfer coefficient ' $h$ ' and heat transfer rate ' $Q$ ' follows the same trend as for the copper plate[16] but with a little lower value because of the low thermal conductivity of aluminum plates as compared to copper plate. It is obvious that ' $h$ ' increases as compared to flat plates because the dimple arrangement causes delay in the development of the thermal boundary layer \& hence increases the local heat transfer in the reattachment region and increases the heat transfer coefficient [1].It can also be seen that ' $Q$ ' increases with decrease in orientation angle of dimples. Because the near-wall turbulent mixing intensity downstream the dimple increases due to the vortex flow shedding from the dimples[6,15].

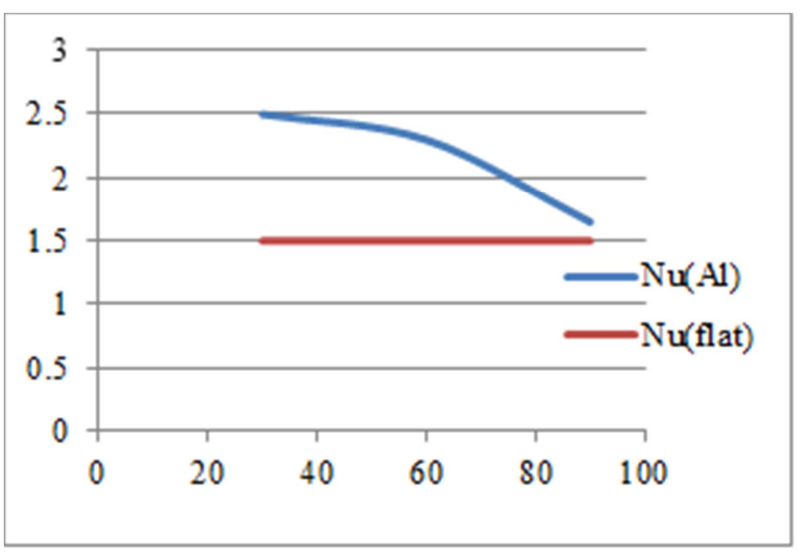

Figure 6. Variation of Nusselt number with orientation of dimples. 


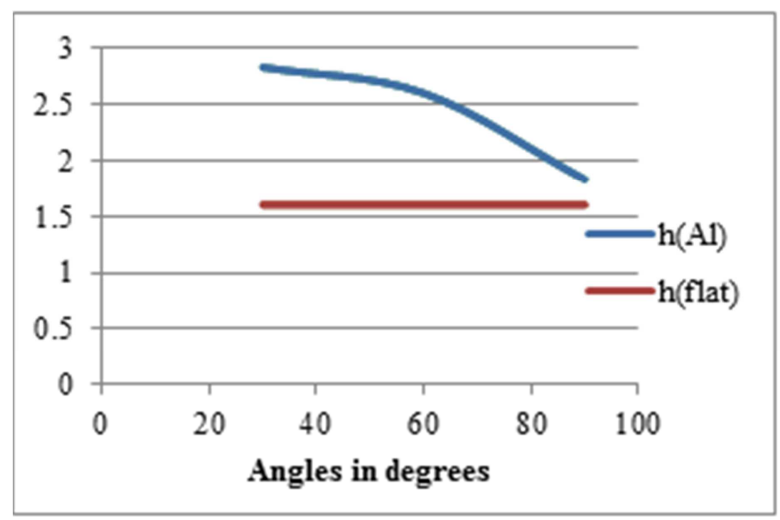

Figure 7. Variation of Heat transfer coefficient with orientation of dimples.

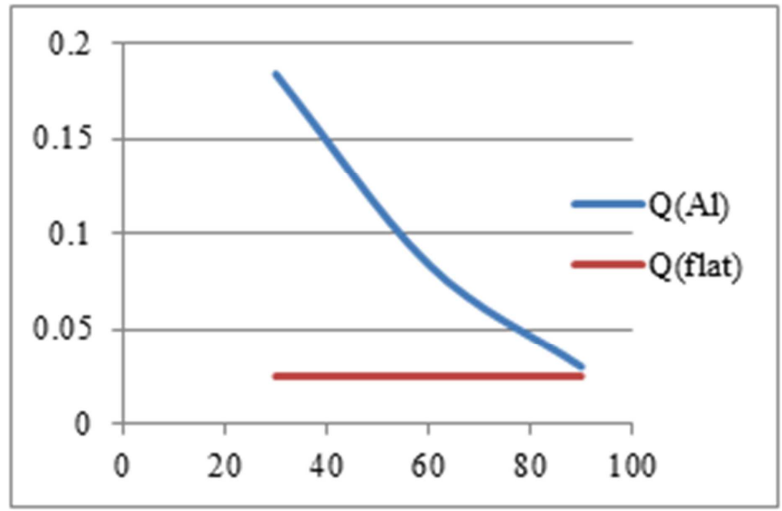

Figure 8. Variation of Heat transfer rate with orientation of dimples.

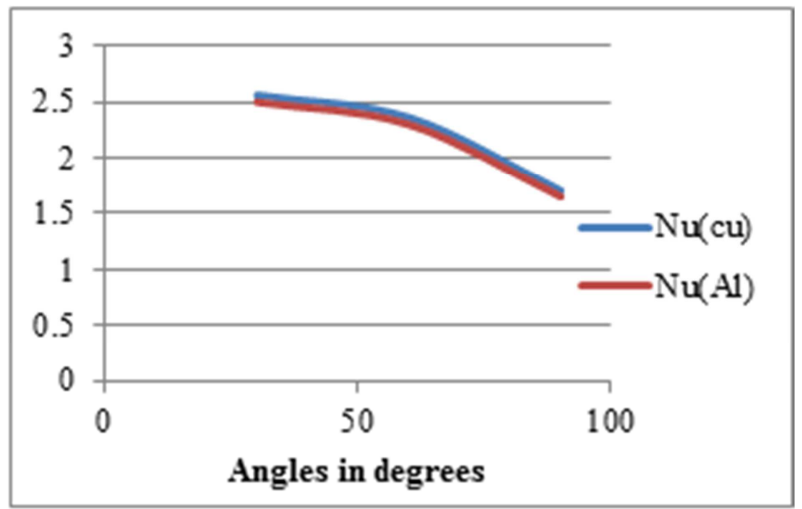

Figure 9. Comparison of Nusselt number.

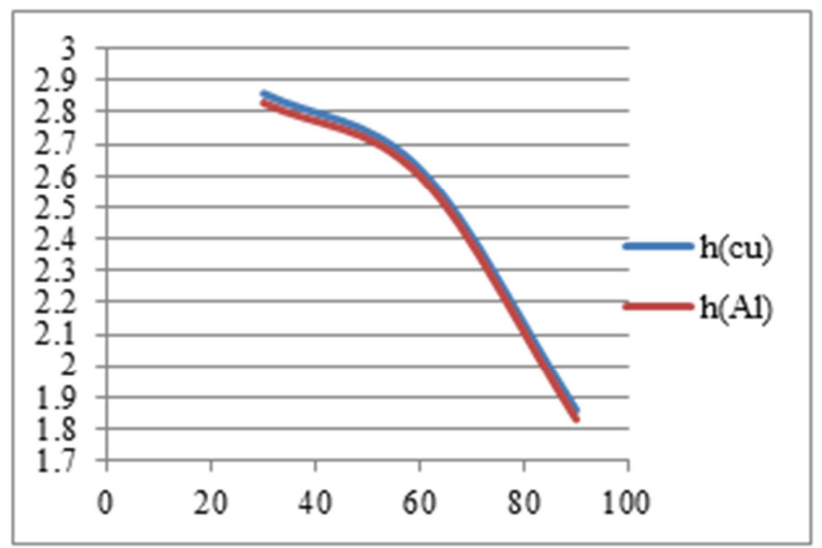

Figure 10. Comparison of Heat transfer coefficient.

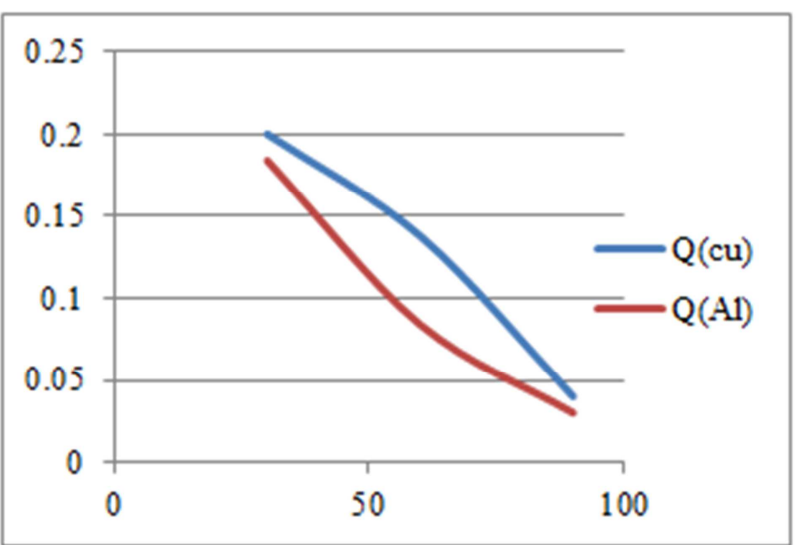

Figure 11. Comparison of Heat transfer rate.

Figure 9, 10, 11 shows Comparison of Nusselt number ' $\mathrm{Nu}$ ', heat transfer coefficient ' $h$ ' and heat transfer rate ' $Q$ ' with different orientation of dimples. It can be seen that both the plates follows the same trend i.e. with decrease in orientation angle the value increase because at low angles $\left(30^{\circ}\right)$ the dimples are close as compared at higher angles $\left(90^{\circ}\right)$.So a pronounce effect of dimples is felt.

\section{Conclusion}

In this experimental work an investigation of the effect of air flow over a flat plate with different orientation of dimples on the flat plate is carried out. The main conclusions of the work are:

- Nusselt number ' $\mathrm{Nu}$ ', and heat transfer coefficient ' $h$ ' increases with different orientation of dimples as compared to the flat plate due to direct flow impingement on the downstream boundary and strengthened flow mixing by the vortices at the downstream.

- It can be seen that as expected the Nusselt number, and heat transfer coefficient ' $h$ ' curve for aluminum plate follows the same trend as for the copper plate[16] but with a little lower value because of the low thermal conductivity of aluminum plates.

- Nusselt number ' $\mathrm{Nu}$ ' and heat transfer coefficient ' $h$ ' are higher for low angle orientation of dimples because at low angles the dimples are close to each other hence a pronounce effect of dimples is felt which is obvious from the figure.

\section{References}

[1] Zhang, D., Zheng, L., Xie, G., and Xie, Y.,An Experimental Study on Heat Transfer enhancement of Non-Newtonian Fluid in a Rectangular Channel with Dimples/Protrusions, Transactions of the ASME, Vol. 136, pp.021005-10, 2014.

[2] Beves, C.C., Barber, T.J., and Leonardi,E., An Investigation of Flow over Two-Dimensional Circular Cavity. In 15th Australasian Fluid Mechanics Conference, the University of Sydney, Australia, pp.13-17, 2004. 
[3] Kuethe A. M., Boundary Layer Control of Flow Separation and Heat Exchange. US Patent No. 1191, 1970.

[4] Afanasyev,V.N.,Chudnovsky,Y.P.,Leontiev,A.I.,andRoganov,P. S., Turbulent flow friction and heat transfer characteristics for spherical cavities on a flat plate. Experimental Thermal Fluid Science, Vol. 7, Issue 1, pp. 1-8, 1993.

[5] Chyu, M.K., Yu, Y., Ding, H., Downs, J.P., and Soechting, F.O., Concavity enhanced heat transfer in an internal cooling passage. In Orlando international Gs Turbine \& Aero engine Congress \& Exhibition, Proceedings of the 1997(ASME paper 97-GT-437), 1997.

[6] Mahmood,G.I.,Hill,M.L.,Nelson,D.L.,Ligrani,P.M.,Moon,H.K ., and Glezer,B., Local heat transfer and flow structure on and above a dimpled surface in a channel. J Turbomach, Vol.123, Issue 1, pp: 115-23, 2001.

[7] Mahmood, G. I., andLigrani, P. M., Heat Transfer in a Dimpled Channel: Combined Influences of Aspect Ratio, Temperature Ratio, Reynolds Number, and Flow Structure. Int. J. Heat Mass Transfer, Vol.45, pp.2011-2020, 2002.

[8] Xie, G. N., Sunden, B., and Zhang, W. H., Comparisons of Pins/Dimples Protrusions Cooling Concepts for an Internal Blade Tip-Wall at High Reynolds Numbers. ASME J. Heat Transfer, Vol.133, Issue 6, pp. 0619021-0619029, 2011.

[9] Gadhave,G., and Kumar.P., Enhancement of forced Convection Heat Transfer over Dimple SurfaceReview.International Multidisciplinary e - Journal .Vol-1, Issue-2, pp.51-57, 2012
[10] Katkhaw, N., Vorayos, N., Kiatsiriroat, T., Khunatorn, Y., Bunturat, D., and Nuntaphan., A. Heat transfer behavior of flat plate having 450 ellipsoidal dimpled surfaces. Case Studies in Thermal Engineering, vol.2, pp. 67-74, 2014

[11] Patel,I.H .,andBorse ,S.H. Experimental investigation of heat transfer enhancement over the dimpled surface. International Journal of Engineering Science and Technology,Vol.4, Issue 6,pp.3666-3672, 2012.

[12] Faheem Akhtar, Abdul Razak R Kaladgi and Mohammed Samee, Heat transfer augmentation using dimples in forced convection -an experimental approach. Int. J. Mech. Eng. \& Rob. Res. Vol4,Issue 1 ,pp 150-153,2015.

[13] Faheem Akhtar, Abdul Razak R Kaladgi and Mohammed Samee, Heat transfer enhancement using dimple surfaces under natural convection - an experimental study, Int. J. Mech. Eng. \& Rob. Res. Vol 4, Issue1, pp 173-175, 2015.

[14] Yu Rao ,Yamin Xu,Chaoyi Wan,A Numerical Study of the Flow and Heat Transfer in the Pin Fin-Dimple Channels With Various Dimple Depths, Journal of Heat Transfer ,Transactions of the ASME,Vol. 134, pp-071902-1-9, 2012.

[15] P.M. Ligrani, J.L. Harrison, G.I. Mahmood, M.L. Hill, Flow structure due to dimple depression on a channel surface, Phys. Fluids 13 (2001) 3442-3451.

[16] Hasibur Rahman Sardar and Abdul Razak Kaladgi ,Forced Convection Heat Transfer Analysis through Dimpled Surfaces with Different Arrangements, American Journal of Energy Engineering Vol 3 ,Issue 3,pp 37-45,2015. 